\title{
Thoracoscopic Pulmonary Lobectomy for Densely Fused Pulmonary Lobes in Children with Congenital Pulmonary Airway Malformation: Technical Tips
}

\author{
Hiroyuki Koga, MD, Hiroki Nakamura, MD, ${ }^{1}$ Hiroshi Murakami, MD, ${ }^{1}$ Shunki Hirayama, MD, ${ }^{2}$ \\ Kota Imashimizu, MD, ${ }^{2}$ Kinya Nishimura, MD, ${ }^{3}$ Kazuhiro Suzuki, MD, ${ }^{4}$ Ryohei Kuwatsuru, MD, \\ Eiichi Inada, MD, ${ }^{3}$ Kenji Suzuki, MD, ${ }^{2}$ and Atsuyuki Yamataka, MD ${ }^{1}$
}

\begin{abstract}
Aim: Thoracoscopic pulmonary lobectomy (TPL) is extremely challenging in cases where severe incomplete fissure causes densely fused pulmonary lobes (DFPL) since pulmonary arteries (PAs) are buried and completely concealed by DFPL. We describe TPL for DFPL including a technical tip to prevent pitfalls.

Materials and Methods: Four congenital pulmonary airway malformation (CPAM) and DFPL (left-upper: 2, left-lower: 1, right-middle: 1) were treated. During TPL, DFPL prevent interlobar PAs from being identified and searching for them only promotes bleeding and air leakage, serious pitfalls that affect the safety and success of TPL. Our tip is to ligate and divide the pulmonary veins (PVs) at the pulmonary hilum and the hilar PA supplying the CPAM lobe to expose the bronchus of the lobe, which is then ligated and divided. The main PA supplying the lobe running underneath the DFPL is exposed and visible from the pulmonary hilum allowing the PA supplying the lobe to be ligated and divided safely. A line demarcating the fused fissure becomes apparent, and an endoscopic stapler or EnSeal ${ }^{\circledR}$ device can be used to divide the DFPL along the line taking great care not to injure the main PA or interlobar PAs.

Results: There were no intra-/postoperative complications in any case. All patients performed well without respiratory tract-related symptoms after a mean follow-up of 4.6 years.

Conclusions: TPL for DFPL in children with CPAM can be performed safely and successfully as a virtually bloodless procedure and without incidence of air leakage by ligating and dividing the PA after dividing the PVs and bronchus to the lobe.
\end{abstract}

Keywords: thoracoscopic surgery, pulmonary lobectomy, densely fused fissure, congenital pulmonary airway malformation

\section{Introduction}

W ITH ADVANCEMENTS IN endoscopic technology and technique, minimally invasive surgery is increasingly recognized as a safe and feasible technique for resecting congenital pulmonary airway malformations (CPAM). Prolonged air leakage after a lobectomy frequently occurs especially in patients with densely fused pulmonary lobes (DFPL). Surgically treating densely fused fissure can prolong operative time, increase blood loss, and cause morbidity such as due to air leakage, which can in turn prolong the duration of chest tube drainage in patients and hospitalization. ${ }^{1}$ For pediatric surgeons, especially during thoracoscopic pulmonary lobectomy (TPL), dealing with densely fused fissure presents difficult problems.

There are two approaches to solve the problem of densely fused fissure: fissure-less technique and retrograde dissection (fissure-first). There have been reports on the fissure-less

\footnotetext{
Departments of ${ }^{1}$ Pediatric General and Urogenital Surgery, ${ }^{2}$ Thoracic General Surgery, ${ }^{3}$ Anesthesiology and Pain Medicine, and ${ }^{4}$ Radiology, Juntendo University School of Medicine, Tokyo, Japan.

This article was presented at the 25th Annual Congress of the International Pediatric Endosurgery Group, April 11-14, 2018, in Seattle, Washington.
} 
technique according to which the final step is usually the division of the parenchyma using staplers. ${ }^{2}$ To the best of our knowledge, there have only been several reports demonstrating the efficacy of thoracoscopic lobectomy for densely fused fissure, ${ }^{3-6}$ although the subjects were not pediatric patients. However, there have been no reports regarding pediatric TPL for DFPL.

Here, we describe TPL for DFPL including a technical tip to prevent possible pitfalls when using it on pediatric patients.

\section{Materials and Methods}

Four CPAM patients (right middle lobe $=1$, left upper lobe $=2$, left lower lobe $=1$ ) with densely fused fissures were treated using our TPL; mean age at surgery was 27.2 months (23.0, 24.9, 25.2, and 36.0 months, respectively) and mean weight at surgery was $11.7 \mathrm{~kg}$ (range $10.0-15.1 \mathrm{~kg}$ ). One of the cases was diagnosed prenatally and two suffered repeated pulmonary infections. All the densely fused fissures were classified as fissural grades III or IV. ${ }^{7}$

\section{Surgical technique}

Our conventional TPL technique is a five-port technique with surgeon and assistant standing facing the patient, as previously described. ${ }^{8}$ The patient is positioned in the lateral decubitus position under general anesthesia with single lung ventilation using the Fogarty catheter. The lateral decubitus position was used because it allows access to the hilum both anteriorly and posteriorly. For thoracoscopy, the surgeon and assistant stand facing the patient while viewing a monitor positioned behind the patient. Initial optical trocar is placed using a closed technique $1 \mathrm{~cm}$ below the inferior angle of the scapula. The closed technique using an optical trocar enables artificial pneumothorax in the best possible way since it prevents against leakage around the trocar site, thus facilitating complete collapse of the lung. The initial trocar defines the position of the fissure and evaluates the general condition of the lung parenchyma. The initial trocar is used for lung retraction. Low flow (0.5-1.0 L/minute) and low pressure (4$6 \mathrm{mmHg}$ ) carbon dioxide was insufflated to collapse the lung. A $5 \mathrm{~mm}$ scope $\left(30^{\circ}\right)$ was used to determine the position of the major fissure and lung parenchyma, and four other working ports were placed to allow optimal access. For lower lobectomy, trocars for the scope, the surgeon's left hand, and the surgeon's right hand are placed in the sixth, fourth, and eighth intercostal spaces in the anterior axillary line, respectively. An extra trocar is placed in the 10th intercostal space in the posterior axillary line to dissect or view vital structures, such as the pulmonary veins (PVs), bronchus, and the feeding arteries from a posterior perspective, as well as to observe the entire pulmonary artery (PA), aortic arch, and course of the vagus nerve. This trocar ensures the safety and reliability of TPL since dissection of the feeding artery or arteries originating from the aorta and dissection of the posterior wall of the inferior PV and left bronchus are facilitated by switching of the scope between the 8th intercostal space trocar and the 6 th intercostal space trocar, the left-hand instrument between the 6th intercostal space trocar and the 4th intercostal space trocar, and the right-hand instrument between the 10th intercostal space trocar and the 8th intercostal space trocar. Switching back and forth also contributes to minimizing blood loss during TPL. For an upper lobectomy, the trocar positions are one intercostal space higher than those used in lower lobectomy. For a middle lobectomy, the trocars for the right hand, the scope, and the left hand are placed in the fourth, fifth, and seventh intercostal spaces in the anterior axillary line, respectively. To create more space between the scope trocar and the right-hand trocar, the scope trocar can be placed slightly more anteriorly than the right-hand trocar, which will prevent the scope and the right-hand instrument from hitting each other.

The case of the right middle lobectomy. Upon close examination, the fissures of the right lung were found to be fused. In particular, the minor fissure was so densely fused that it was totally undistinguishable. First, the vein of the right middle lobe, which drains into the superior $\mathrm{PV}$, was isolated and divided using clips such as Endo-clips ${ }^{\mathrm{TM}}$ (Tyco Healthcare, CT) or Hem-o-lok ${ }^{\mathrm{TM}}$ clips (Teleflex Medical, NC). The bronchus was then exposed, encircled with a thick silk tie as a traction suture, and divided using Endo-clips by applying traction and counter traction on the silk tie. Finally, the arteries of the right middle lobe were identified and ligated using Endo-clips. By doing so, a line demarcating the major fissure could be identified and dissected using a stapling device, Echelon-flex ${ }^{\mathrm{TM}}$ (Ethicon Endo-surgery; Johnson \& Johnson, Cincinnati, $\mathrm{OH}$ ), which was used to seal the lung parenchyma and create a division between the right upper and middle lobes.

The case of the left upper lobectomy. Mediastinal A3 and the superior PV at the pulmonary hilum were identified. Mediastinal A3 was mobilized and dissected free taking great care not to injure as it originates proximally from the PA and is short pedicled. We strongly recommend dividing mediastinal V1+2 and V3 of the superior PV first and then dissecting the anterior aspect of the upper lobe bronchus to expose mediastinal A3 fully. Then, clip, seal, and divide A3. During mobilization and dissection of V1 +2 and V3, keep in mind that the posterior wall of the superior PV is closely attached to the bronchus to the upper lobe. The bronchus to the upper lobe is mobilized, cleaned, and divided with a stapler (Fig. 1A). Keep in mind that the posterior wall of the upper lobe bronchus is close to the PA. The interlobular PA and its branches will be exposed by dissecting the PA along with its wall beneath the PA sheath. Figure 1B shows the divided interlobular A4 and A5. Following the division of both basal and superior segmental arteries, the fissure is divided using a stapler (Fig. 1C, D).

The case of left lower lobectomy. To divide a densely fused fissure, the interlobular PA must be exposed. The pulmonary parenchyma constituting the densely fused fissure overlaying A8-10 is usually thin. Thus, A8 was exposed by incising the pleura and the thin parenchyma using electrocautery, a tunnel was created toward the posterior mediastinum by dissecting the PA along with its wall beneath the PA sheath, taking great care not to injure the patient's A6 and $A 1+2$. Then, the fussed fissure was safely divided toward the posterior aspect using EnSeal ${ }^{\circledR}$ (Ethicon Endo Surgery, Inc., Cincinnati, $\mathrm{OH})$. Finally, a pair of forceps was passed from anterior to posterior after A6 was identified, and the fissure division was completed. After completion of the fissure, the 

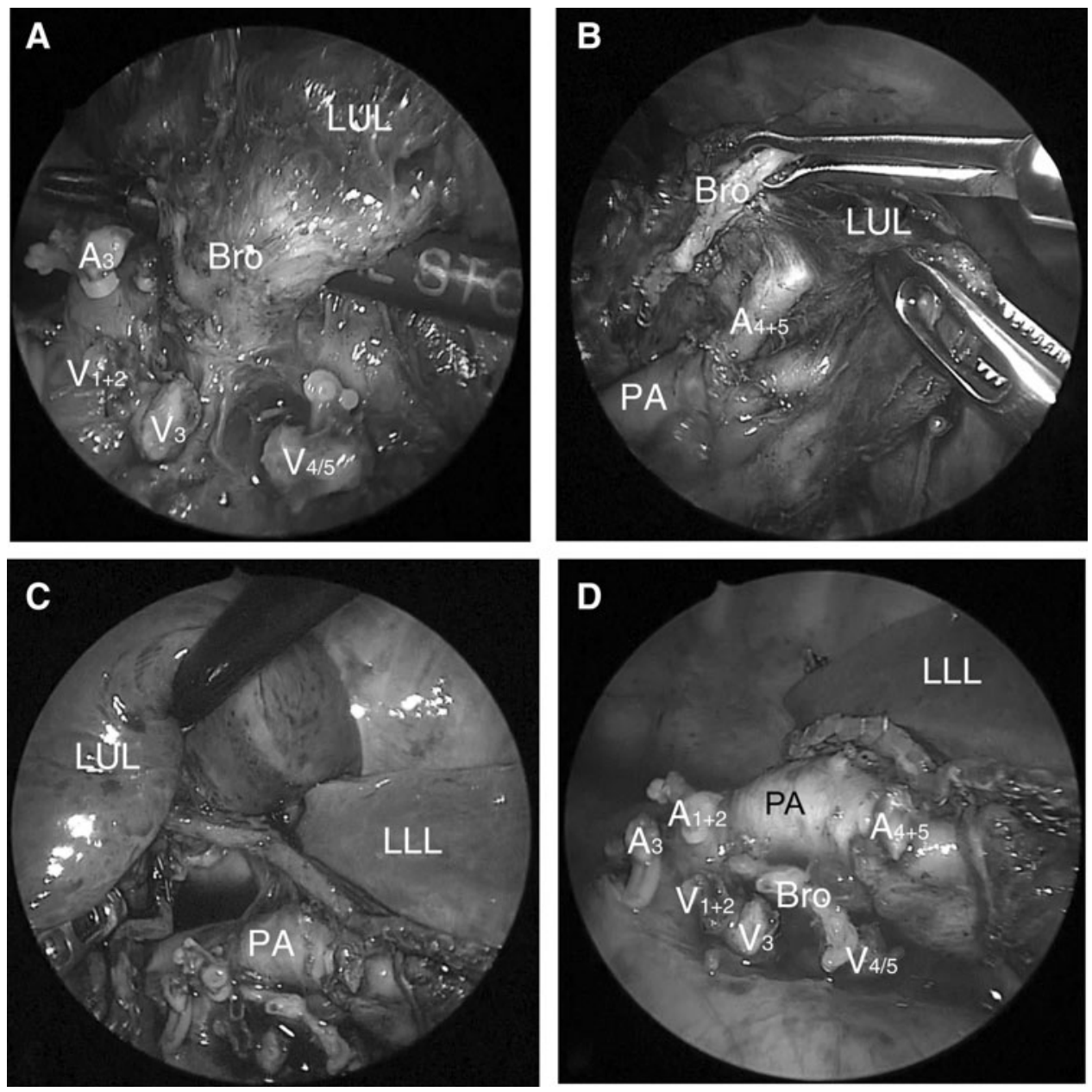

FIG. 1. Left upper lobectomy. (A) After mediastinal A3, V1+2, V3, and V4/5 are sealed and divided, the Bro to the upper lobe is mobilized cleaned, and divided with a stapler. (B) The interlobular PA $(\mathrm{A} 4+5)$ and its branches are exposed by dissecting the PA along with its wall beneath the PA sheath. (C, D) Following the division of both basal and superior segmental arteries, the fissure is divided using a stapler. Bro, bronchus; LLL, left lower lobe; LUL, left upper lobe, PA, pulmonary artery.

PV and the bronchus were divided using a surgical stapler, thereby completing the lobectomy.

One of the incisions was extended to $\sim 2.0 \mathrm{~cm}$ to facilitate the removal of the resected lobe from the thoracic cavity. Fibrin glue (Bolheal ${ }^{\circledR}$; Chemo-Sero-Therapeutic Research Institute, Kumamoto, Japan) and thick polyglycolic acid mash (Neoveil ${ }^{\circledR}$ Gunze Corp Ltd., Kyoto, Japan) were used as tissue sealants to prevent air leakage. At the end of the procedure, a water-submersion test was used to detect the presence of air leaks. Distilled water was instilled into the chest cavity and the lung was reinflated to a pressure of $20 \mathrm{cmH}_{2} \mathrm{O}$.

\section{Postoperative care}

The tube was placed on $10 \mathrm{cmH}_{2} \mathrm{O}$ suction and left in situ overnight. If there was no air leakage after the surgery, the chest tube was water-sealed the next morning and a chest $\mathrm{X}$-ray radiography (CXR) was taken after 6 hours. If the CXR was normal, the tube was clamped, and another CXR was taken after 6 hours. If the CXR was again normal, the tube was removed. A cephalosporin antibiotic was administered during the 24 hours postoperatively. Pain was usually controlled adequately by an intravenous narcotic used for 24-36 hours postoperatively. A regular diet was resumed on the day of surgery or from the morning after the surgery. Patients were ready for discharge from the hospital usually on days 2 or 3 after TPL where air leakage was not present.

\section{Results}

The outcomes were successful in all four cases, without any intraoperative complications and no need for switching to thoracotomy. The mean duration of surgery was 310 minutes (range 220-390 minutes). The mean operative blood loss was minimal (range 1-5 mL). No postoperative complications were encountered, such as air leakage. Chest tubes were removed by the second postoperative day in all cases. All patients performed well until the present without respiratory tract-related symptoms after a mean follow-up of 4.6 years (range $0.7-8.2$ years). 


\section{Discussion}

The incidence of prolonged air leakage following pulmonary surgery is reported in $8 \%-15 \%$ of cases in different studies, and it leads to increased length of hospital stay, higher overall complication rates, and higher hospital costs. ${ }^{9-12}$ Dissection through the fissure is well known to increase the incidence of prolonged air leakage especially in patients with densely fused fissures. ${ }^{13,14}$ The surgical approach into the fissural parenchyma may be an important and modifiable factor for the prevention of possible air leakage. ${ }^{15}$ To avoid severe complications, our tips are to ligate and divide the PVs at the pulmonary hilum and the hilar PA supplying the CPAM lobe to expose the bronchus of the CPAM lobe, which is then ligated and divided. The main interlobar PA supplying the CPAM lobe running underneath the DFPL is exposed and thus made visible from the pulmonary hilum allowing the interlobular PA supplying the lobe to be ligated and divided safely. A line demarcating the fused fissure becomes apparent and an endoscopic stapler or EnSeal device can be used to divide the DFPL along the line exercising great caution not to injure the main interlobar PA or interlobar PAs.

Several reports have described the efficacy of fissure-less lobectomy since Temes et al. first reported fissure-less lobectomy as a procedure to avoid postoperative prolonged air leakage. ${ }^{2}$ In a report which included resection on all lobes, Gómez-Caro et al. reported that the use of a fissure-less technique significantly reduced the incidence and duration of air leakage when compared with conventional techniques, as well as higher probability of cessation of air leaks without an increase in morbidity and mortality. ${ }^{13}$ Similarly, $\mathrm{Ng}$ et al. found that the use of a fissure-less technique reduced the duration of chest tube drainage and shortened the length of hospital stay. ${ }^{14}$ Stamenovic et al. reported about thoracoscopic fissure-less lobectomy, which was significantly superior to the conventional TPL regarding the occurrence rate of prolonged air leakage, the duration of chest tube drainage, and the length of hospital stays. ${ }^{6}$ Therefore, Stamenovic et al. concluded that a thoracoscopic fissure-less lobectomy appears to be a superior technique to conventional TPL in terms of preventing prolonged air leakage (PAL) and reducing the length of hospital stay. Indeed, our series shows that thoracoscopic fissure-less lobectomy, even though performed in pediatric patients, prevents PAL and reduces the amount of time for keeping the chest tube inserted.

In fissure-less lobectomies, the dominant bronchus is usually resected before the major PAs are resected. Therefore, the procedure sometimes appears to be unsafe for surgeons who are not familiar with it. Especially in either of the lower lobectomy cases, the PA that needed resecting is located right behind the lower bronchus, which could have potentially lead to a catastrophic pulmonary arterial injury when the connective tissue between the lower bronchus and the PA was dissected. To avoid this pulmonary arterial injury, we performed retrograde approach (fissure-first) for treating densely fused lower lobectomy. Once A8 of the PA was exposed, a tunnel was created toward the posterior mediastinum by dissecting the sheath of the PA along with its wall beneath the PA sheath. We believe that using this technique was crucially important to avoid an accidental injury of the pulmonary arterial wall in the case of either lower lobectomy.
Another way would be to place an extra trocar, we placed our thoracoscopic port at the 10th intercostal space in the posterior axially line, and this gave us an operative view with superior direct vision during dissection from a posterior perspective as well as possibility to observe the entire PA, aortic arch, and course of the vagus nerve, which we strongly are convinced ensures the safety and reliability of TPL. This thoracoscopic surgery technique is useful for avoiding postoperative prolonged air leakage in cases where densely fused fissure is present.

In this series, hemorrhage was not observed in any of the patients with densely fused fissure. TPL for densely fused fissure requires a high degree of anatomical knowledge to prevent hemorrhage from happening. Preoperative computerized tomography imaging was employed to assess for certain important keys: (1) the presence of a common trunk of the PV, (2) the presence of the lingular PA branching from A8, and (3) the distance between the branches of A1 +2 and A6. When the distance between the branches of $\mathrm{A} 1+2$ and A6 is short, great caution has to be exercised when the ligation is passed from anterior to posterior. The anatomy of the pulmonary structures must be understood and any variations or anomalies must be delineated preoperatively.

\section{Conclusion}

TPL applied to DFPL in pediatric patients where CPAM is present can be performed safely and with great results, that is, as a virtually bloodless procedure air leakage free by way of ligating and dividing the interlobular PA after dividing the PVs and bronchus to the CPAM lobe.

\section{Disclosure Statement}

No competing financial interests exist.

\section{References}

1. Stolz AJ, Schutzner J, Lischke R, Simonek J, Pafko P. Predictors of prolonged air leak following pulmonary lobectomy. Eur J Cardiothorac Surg 2005;27:334-336.

2. Temes RT, Willms CD, Endara SA, Wernly JA. Fissureless lobectomy. Ann Thorac Surg 1998;65:282-284.

3. Decaluwe H, Sokolow Y, Deryck F, Stanzi A, Depypere L, Moons J, et al. Thoracoscopic tunnel technique for anatomical lung resections: A "fissure first, hilum last" approach with staplers in the fissureless patient. Interact Cardiovasc Thorac Surg 2015;21:2-7.

4. Igai H, Kamiyoshihara M, Yoshikawa R, Osawa F, Kawatani N, Ibe T, et al. The efficacy of thoracoscopic fissureless lobectomy in patients with dense fissures. J Thorac Dis 2016;8:3691-3696.

5. Samejima J, Mun M, Matsuura Y, Nakao M, Uehara H, Nakagawa K, et al. Thoracoscopic anterior "fissure first" technique for left lung cancer with an incomplete fissure. J Thorac Dis 2016;8:3105-3111.

6. Stamenovic D, Bostanci K, Messerschmidt A, Jahn T, Schneider T. Fissureless fissure-last video-assisted thoracoscopic lobectomy for all lung lobes: A better alternative to decrease the incidence of prolonged air leak? Eur $\mathbf{J}$ Cardiothorac Surg 2016;50:118-123.

7. Craig SR, Walker WS. A proposed anatomical classification of the pulmonary fissures. J R Coll Surg Edinb 1997; 42:233-234. 
8. Yamataka A, Koga H, Ochi T, Imashimizu K, Suzuki K, Kuwatsuru R, et al. Pulmonary lobectomy techniques in infants and children. Pediatr Surg Int 2017;33:483-495.

9. Allen MS, Darling GE, Pechet TT, Mitchell JD, Herndon JE, 2nd, Landreneau RJ, et al. Morbidity and mortality of major pulmonary resections in patients with early-stage lung cancer: Initial results of the randomized, prospective ACOSOG Z0030 trial. Ann Thorac Surg 2006;81:10131019; discussion 9-20.

10. Brunelli A, Cassivi SD, Halgren L. Risk factors for prolonged air leak after pulmonary resection. Thorac Surg Clin 2010;20:359-364.

11. Cerfolio RJ. Air leaks and the pleural space. Chest Surg Clin N Am 2002;12:ix-x.

12. Varela G, Jimenez MF, Novoa N, Aranda JL. Estimating hospital costs attributable to prolonged air leak in pulmonary lobectomy. Eur J Cardiothorac Surg 2005;27:329-333.

13. Gómez-Caro A, Calvo MJ, Lanzas JT, Chau R, Cascales P, Parrilla P. The approach of fused fissures with fissureless technique decreases the incidence of persistent air leak after lobectomy. Eur J Cardiothorac Surg 2007;31:203-208.

14. Ng T, Ryder BA, Machan JT, Cioffi WG. Decreasing the incidence of prolonged air leak after right upper lobectomy with the anterior fissureless technique. J Thorac Cardiovasc Surg 2010;139:1007-1011.

15. Okereke I, Murthy SC, Alster JM, Blackstone EH, Rice TW. Characterization and importance of air leak after lobectomy. Ann Thorac Surg 2005;79:1167-1173.

Address correspondence to:

Hiroyuki Koga, MD

Department of Pediatric General and Urogenital Surgery Juntendo University School of Medicine 2-1-1 Bunkyo-ku Tokyo 113-8421

Japan

E-mail: h-koga@juntendo.ac.jp 